\title{
Space Charge Behaviours in Polyethylene under Combined AC and DC Electric Fields
}

\author{
C. Zhou and G. Chen \\ The Tony Davies High Voltage Laboratory, University of Southampton \\ Southampton SO17 1BJ, United Kingdom \\ *E-mail: cz3g13@ecs.soton.ac.uk
}

\begin{abstract}
Polyethylene has been one of the widely studied polymeric insulation materials. One of the major issues related to polymeric materials is the easy formation of space charge which may cause electric field enhancement. In this paper, a numerical simulation based on a bipolar charge injection/transport model is used to obtain characteristics of space charge in polyethylene under the combined AC and DC high voltage at room temperature. The bipolar charge injection/transport model, which is widely used in HVDC space charge simulation, is applied in this combined condition. The overall applied voltage, consisted of root mean square (RMS) values of $50 \mathrm{~Hz} \mathrm{AC}$ voltage and DC voltage, is kept the same, while the DC component has been varied from 0 to 1 . The simulated charge distributions present notable differences when DC offset is added compared with pure AC conditions. Besides, these differences become more significant when the offset ratio is increased. The total positive and negative charge amounts are calculated respectively by integrating the charge in the material, and a curve of net charge amount changing along with time is obtained.
\end{abstract}

\section{INTRODUCTION}

Over years, space charge accumulation has been regarded as an important factor causing the electrical failure of high voltage DC insulation. Those accumulated charges can distort the internal electric field in return. This distortion could be exacerbated by the mutual effects of mechanical and thermal stress existed from either the applied electric field or the local environment. Over the last few decades, several nondestructive charge mapping methods have been established to investigate the formation, transportation, and neutralization of space charge within polymeric materials [1,2]. They provide inspiring understanding on charge injection, transportation, trapping, detrapping and recombination in insulation materials. Besides, considerable efforts have been made to study space charge characteristics in polymeric insulation under dc conditions [3-5]. However, space charge behaviour under ac voltages and combined conditions, which are common situation in high voltage convertor transformers, has not been fully investigated. This paper mainly focuses on numerical modelling of space charge under combined AC and DC high voltage conditions. Polyethylene, especially low density type (LDPE), is chosen as the research material in order to simplify the simulated situations and minimise influence factors.

Two possible reasons are account for the current limited research on space charge under complex electric stresses. Firstly, previous experimental measurements showed small amount of space charge gathered under AC electric fields, and the accumulated amount was significantly reduced under high frequency conditions [6, 7]. Therefore, space charge under complex electric stresses is assumed to be less harmful than that under a DC field and attracts much less research attention. Secondly, the detection of space charge under complex stresses is relatively difficult comparing with measurement under DC fields. New approaches are required to deal with large amount of data and phase identification.

Numerical modelling provides an alternative method to investigate charge dynamics within solid insulation. A bipolar charge transport model involving bipolar charge injection, transport, trapping, and recombination has been established to analyse the dynamics of space charge within solid dielectrics under DC high electric fields [8,9]. The simulation can represent both reasonable amount of charges and evolution of space charge in the transient process as well as the effect of long term stressing [10]. In this paper, the model is further developed to facilitate the simulation of space charge within polyethylene under combined AC and DC fields with various direct and alternative voltage components' ratios.

\section{BIPOLAR CHARgE TRANSPORT MODEL}

The bipolar charge transport model is assuming that opposite charges contribute to charge injection, transport, and trapping in the bulk of insulator as well as recombination process, when subjected to electric fields [3]. The model has been implemented to simulate space charge within LDPE films which have been subdivided into small elements and the definition of the carrier fluxes between elements as shown in Fig. 1. An unbalanced partition is chosen, in consideration of the charges primarily accumulated in the region close to the interface when the applied field constantly alters its polarity. These settings of the parameters will generate a packet-like charge distribution curves under relatively high HVDC electric fields [11].

All the charges are assumed to be sourced by the Schottky injection from the boundaries, as Eq. (1) shows:

$$
\begin{aligned}
& J_{e}(0, t)=A T^{2} \exp \left(\frac{-e w_{e i}}{\mathrm{kT}}\right) \exp \left(\frac{e}{\mathrm{kT}} \sqrt{\frac{e E(0, t)}{4 \pi \varepsilon}}\right) \\
& J_{h}(d, t)=A T^{2} \exp \left(\frac{-e w_{h i}}{\mathrm{kT}}\right) \exp \left(\frac{e}{\mathrm{kT}} \sqrt{\frac{e E(d, t)}{4 \pi \varepsilon}}\right)
\end{aligned}
$$

Where $\mathrm{J}_{\mathrm{e}}(0, \mathrm{t})$ and $\mathrm{J}_{\mathrm{h}}(\mathrm{d}, \mathrm{t})$ are the injected current density of the electrons at the cathode and of the holes at the anode respectively; $\mathrm{E}(0, \mathrm{t})$ and $E(d, t)$ are the electric fields accordingly; A is the Richardson constant, normally $1.20 \times 10^{6}$ $\mathrm{Am}^{-2} \mathrm{~K}^{-2} ; \mathrm{T}$ is the absolute temperature; $\mathrm{e}$ is the elementary 
charge; $\mathrm{k}$ is the Boltzmann constant, $1.38 \times 10^{-23} \mathrm{~m}^{2} \mathrm{~kg} \mathrm{~s}^{-2} \mathrm{~K}^{-1}$; $w e i$ and $w h i$ are the injection barrier heights for electrons and holes; and $\varepsilon$ is the permittivity of the dielectric. While the extraction of charge from the electrodes can be calculated using $\mathrm{Eq}(2)$ as below:

$$
\begin{aligned}
& J_{e}(d, t)=\mu_{e} E(d, t) n_{e \mu}(d, t) \\
& J_{h}(0, t)=\mu_{h} E(0, t) n_{h \mu}(0, t)
\end{aligned}
$$

Where $J_{e}(d, t)$ and $J_{h}(0, t)$ are the extracted flux of electrons and holes from the electrodes; $\mu$ is the mobility of charge carriers and $\mathrm{n}_{\mathrm{e} \mu}$ and $\mathrm{n}_{\mathrm{h} \mu}$ are the density of mobile electrons and holes respectively.

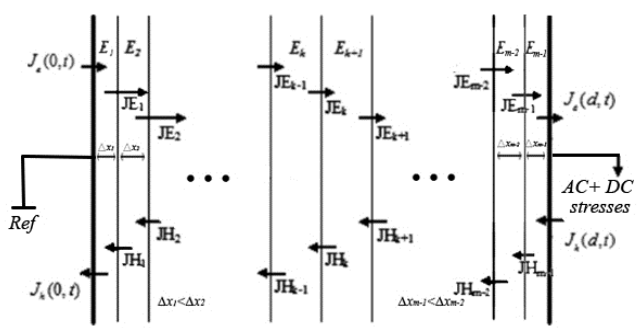

Fig. 1 Discretization of modelled sample under combined AC and DC stress

The charge transport is described by the conduction process characterised using the mobility of the charge carriers. The mobility of the electrons and holes are computed differently in consideration of their different field dependences [12]. The mobility of electrons is analysed using the power's law [13] while that of holes is predicted utilising a curve-fitting function of Fig. 2.(a) [14]. The specific process is illustrated below:

$$
\begin{gathered}
J=\mu n E=v n \\
v=\mu_{0} E^{m_{1}} \quad \text { (Electrons) } \\
v=\left\{\begin{array}{c}
a_{1} E+a_{2} E^{2}+a_{3} E^{3}\left(0<E \leq E_{c}\right) \\
b E^{-m_{2}}\left(E>E_{c}\right)
\end{array} \quad\right. \text { (Holes) }
\end{gathered}
$$

Where $J$ is the conduction current density; $\mu$ is the mobility of charge carrier and is field dependent; $v$ is the drift velocity; $\mu_{0}$ is the mobility under low electric fields; $m_{1}, m_{2}$ are the power indexes; $\mathrm{n}$ is the density of mobile species; and $\mathrm{E}$ is the local electric field; $\mathrm{a}_{1}, \mathrm{a}_{2}, \mathrm{a} 3, \mathrm{~b}$ and $m_{2}$ are the constants obtained from curve-fitting of Fig. 2.(a) $\left(a_{1}\right.$ is $1.746 \times 10^{-14}, a_{2}$ is $-3.141 \times 10^{-22}$, a3 is $1.454 \times 10^{-30}, \mathrm{~b}$ is 19.51 and $m_{2}$ is 1.065 ); Ec is a critical field distinguishing the low and high electric field phenomena for positive charge carriers. In Fig. 2 (a), its value is $100 \mathrm{kV} / \mathrm{mm}$.

When charges move within the bulk, they can be captured by the trap centres originating from physical/chemical defects. We assume that the polymer only contains two types of traps, shallow traps and deep traps. And charges in shallow traps are able to detrap, becoming movable, while those in deep traps cannot.

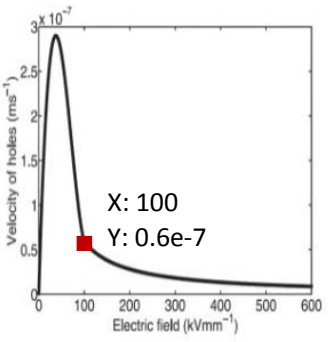

(a)

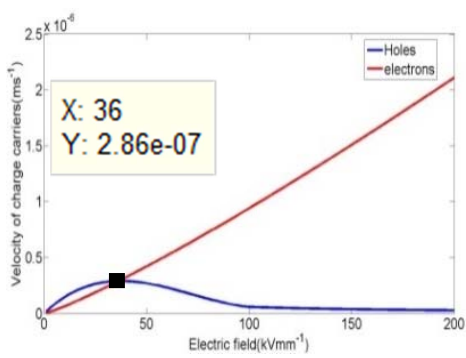

(b)
Fig. 2 Measured holes' velocity versus fields and used velocities versus fields (a) is measured results of Chen [13] and (b) is the used velocities of holes and electrons in the simulation

Theoretically, charge carriers within solids are governed by three basic equations, Poisson's equation Eq(4), Transport equation $\mathrm{Eq}(5)$ and Continuity equation $\mathrm{Eq}(6)$, describing the charge density, current density and electric fields as a function of time and spatial position.

$$
\begin{gathered}
\frac{\partial E(x, t)}{\partial x}=\frac{\rho(x, t)}{\varepsilon} \\
J(x, t) \stackrel{=}{=} \mu n(x, t) E(x, t) \\
\frac{\partial n(x, t)}{\partial t}+\frac{\partial J(x, t)}{\partial x}=s
\end{gathered}
$$

Where $\rho$ is the net charge density; $\varepsilon$ is the permittivity of the dielectric; and $s$ is the source term.

The continuity equation can be split into two, $\operatorname{Eq}(7)$ and $\mathrm{Eq}(8)$. And one is only controlled by the conduction current density, while the other is using the contribution of source term $s$ to update the charge density in the previous one.

$$
\begin{gathered}
\frac{\partial n(x, t)}{\partial t}+\frac{\partial J(x, t)}{\partial x}=0 \\
\frac{\partial n(x, t)}{\partial t}=s
\end{gathered}
$$

The charge trapping and recombination effects are included in the source term $s$ and specific relationships are illustrated in Fig. $3 S_{0}, S_{1}, S_{2}$, and $S_{3}$ are the recombination coefficients for different opposite species; $B_{e}$ and $B_{h}$ are the electrons/holes trapping coefficients.

The total current density $J_{t}(x, t)$ can be computed using $\mathrm{Eq}(9)$ and the first term is the conduction current density and second is the displacement current density.

$$
J_{t}(x, t)=J(x, t)+\varepsilon \frac{\partial E(x, t)}{\partial t}
$$

The applied voltage used in the simulation can be expressed as:

$$
V=V_{P} \sin (2 \pi f t)+V_{\text {offsets }}
$$

Where $V_{p}$ is the peak voltage and $f$ is the frequency AC voltage.

Different parameter settings have been used for electrons and holes respectively for this simulation. The injection barrier for holes is set lower than the electrons in order to demonstrate the phenomenon holes are easier to inject into the insulation, comparing with electrons. All the parameter values are chosen in accordance with those used under purely AC and DC conditions [11,15]. However the mobility of holes is calculated using a curve-fitting function. Specific parameter values are listed in Table I. 


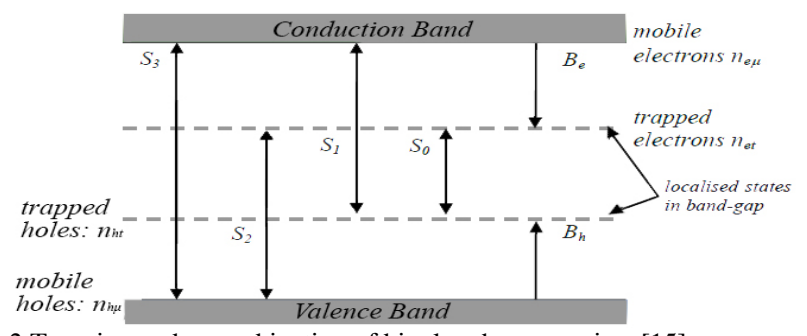

Fig.3 Trapping and recombination of bipolar charge carriers [15]

TABLE I

Parameters for space charge under combined AC and DC simulation [11, 15]

\begin{tabular}{|l|l|l|}
\hline Parameter & Value & Unit \\
\hline Sample thickness & 180 & $\mathrm{um}$ \\
\hline Barrier height for injection & 1.18 & $\mathrm{eV}$ \\
\hline $\mathrm{w}_{\mathrm{ei}}$ (electrons) & 1.16 & $\mathrm{eV}$ \\
\hline $\mathrm{w}_{\text {hi }}$ (holes) & $4.5 \times 10-16$ & $\mathrm{~m}^{2} \mathrm{~V}^{-1} \mathrm{~s}^{-1}$ \\
\hline Low field Mobility $\mu_{0}$ & $\mathrm{~m}^{2} \mathrm{~V}^{-1} \mathrm{~s}^{-1}$ \\
\hline Power law's index of mobility n & 1.165 & $\mathrm{Cm}^{-3}$ \\
\hline Trap density & 100 & $\mathrm{Cm}^{-3}$ \\
\hline $\mathrm{N}_{\text {Oet }}$ (electrons) & 10 & $\mathrm{~s}^{-1}$ \\
\hline $\mathrm{N}_{\text {Oht }}$ (holes) & $7 \times 10-3$ & $\mathrm{~s}^{-1}$ \\
\hline Trapping coefficients & $7 \times 10-5$ & $\mathrm{~m}^{3} \mathrm{C}^{-1} \mathrm{~s}^{-1}$ \\
\hline $\mathrm{B}_{\mathrm{e}}$ (electrons) & $\mathrm{m}^{3} \mathrm{C}^{-1} \mathrm{~s}^{-1}$ \\
\hline $\mathrm{B}_{\mathrm{h}}$ (holes) & $\mathrm{m}^{3} \mathrm{C}^{-1} \mathrm{~s}^{-1}$ \\
\hline Recombination coefficients & $\mathrm{m}^{3} \mathrm{C}^{-1} \mathrm{~s}^{-1}$ \\
\hline $\mathrm{S}_{0}$ trapped electron-trapped hole & $4 \times 10-3$ & \\
\hline $\mathrm{S}_{1}$ mobile electron-trapped hole & $4 \times 10-3$ & \\
\hline $\mathrm{S}_{2}$ trapped electron-mobile hole & $4 \times 10-3$ & \\
\hline $\mathrm{S}_{3}$ mobile electron-mobile hole & 0 &
\end{tabular}

\section{Simulation Results}

For easy comparison, all the simulations have been carried out under the condition of fixed total voltage $9 \mathrm{kV}\left(\mathrm{AC}_{\mathrm{RMS}}+\right.$ DC) as shown in Fig.4. In order to describe the percentage of DC component within the combined electric stress, a DC offset ratio is defined as:

$$
D C \text { off set's ratio }=\frac{V_{D C}}{V_{A C_{-} \mathrm{RMS}}+V_{D C}}
$$

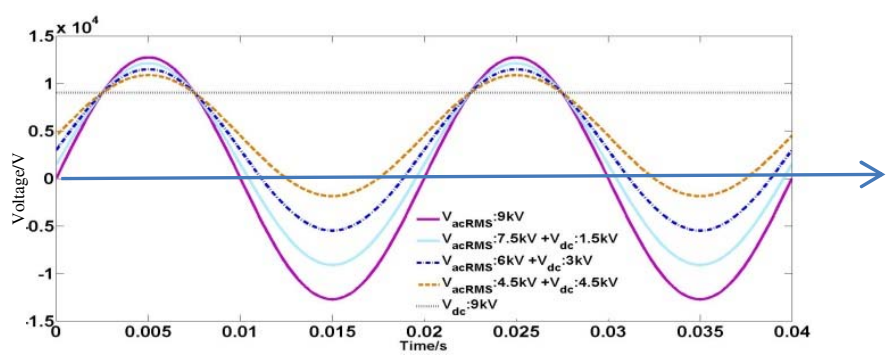

Fig.4 The first two cycles of the applied electric voltages

The simulated charge distribution curves are presented in Fig.5 to Fig.9. The time step used for simulation is $0.002 \mathrm{~s}$, and all the charge profiles under $\mathrm{AC}$ and combined $\mathrm{AC}$ and $\mathrm{DC}$ stresses are plotting the phase angle zero of the applied fields.

Fig.5 is the charge distribution curve under purely AC conditions, and we can see a positive charge accumulation at both electrodes, due to holes' lower injection barrier and smaller mobility. Because more holes are injected at both electrodes during each half cycle and transport slower within the sample, the positive charge will be accumulated near two electrodes. However, the electrons, although in a small amount, can move further into the bulk area. With time, the amount of accumulated charges becomes larger, and the front edges of the two kinds of charge carriers move further.

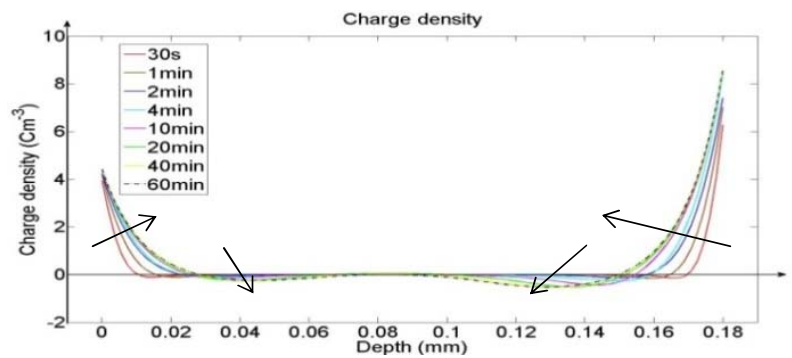

Fig. 5 Charge distribution under $9 \mathrm{kV}$ (RMS) $50 \mathrm{~Hz}$ purely AC stress

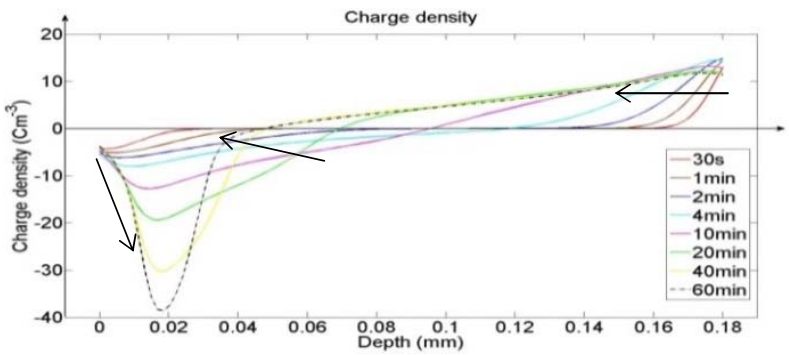

Fig.6 Charge distribution under $7.5 \mathrm{kV}$ (RMS) $50 \mathrm{~Hz}$ AC $+1.5 \mathrm{kV}$ DC stress

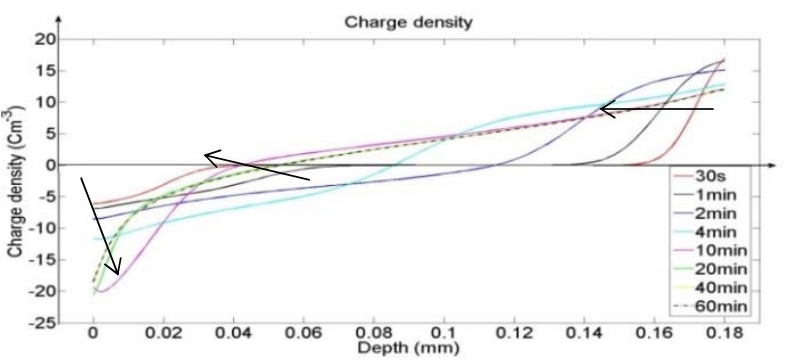

Fig.7 Charge distribution under $6 \mathrm{kV}$ (RMS) $50 \mathrm{~Hz} \mathrm{AC}+3 \mathrm{kV}$ DC stress

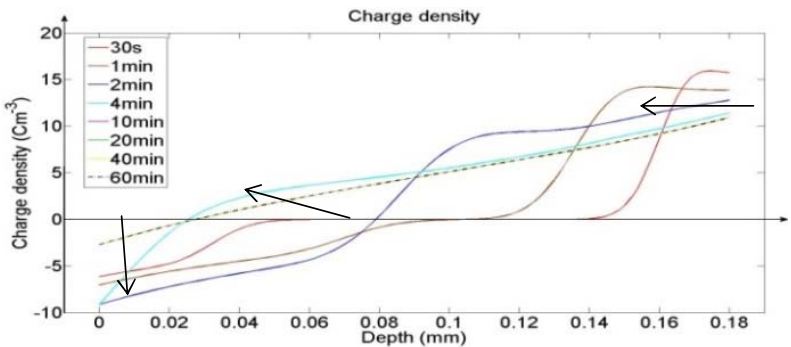

Fig. 8 Charge distribution under $4.5 \mathrm{kV}$ (RMS) $50 \mathrm{~Hz}$ AC $+4.5 \mathrm{kV}$ DC stress

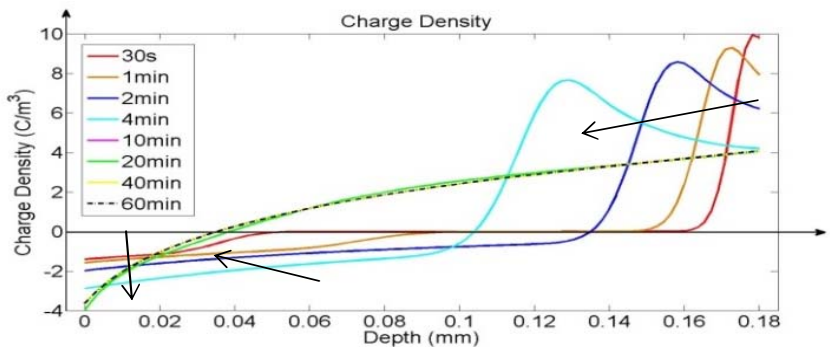

Fig. 9 Charge distribution under $9 \mathrm{kV}$ DC stress 
From Fig.5 to Fig.9, it has been noticed that the charge distribution curves of the purely $\mathrm{AC}$ and the combined stresses are significantly different. And the shapes of the charge distribution curves are much more like a distribution curve under purely DC conditions when the DC offset's ratio is relatively high $(\geq 0.33)$.

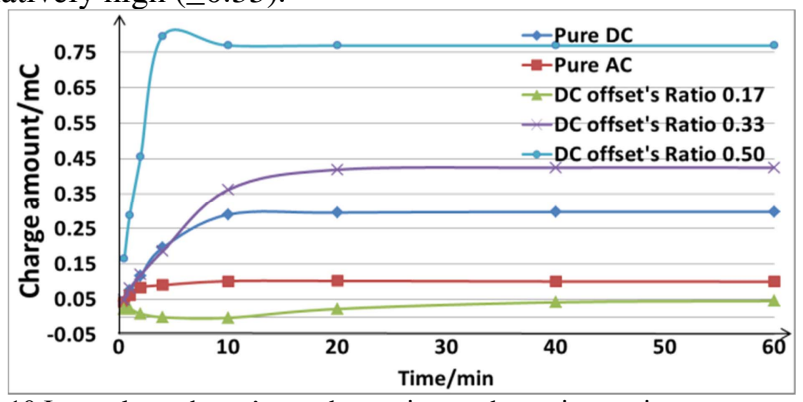

Fig.10 Integral net charge's trend over time under various ratios

Besides, the charge amount accumulated under AC electric stress is smaller than that under the combined conditions, comparing Fig.5 with Fig. 6 to Fig. 8. This is because the DC offsets cause an unsymmetrical time of positive and negative charge injections, like shown in Fig.4, which will enhance the net charge injections. And a large amount of charge will be injected at the above $9 \mathrm{kV}$ (RMS value of the applied fields) as Schottky injection indicates the injected charge amount has an exponential relationship with the electric fields. This can explain the reason why the calculated accumulated charge amount under the combined conditions with high DC offsets' ratio is positive and larger than that under the purely DC conditions as shown in Fig.10.

Furthermore, a negative charge peak can be observed at charge distribution curves under combined conditions. The reason for this is that a larger amount of charges can remain and transport into the bulk of the insulator under the combined $\mathrm{AC}$ and $\mathrm{DC}$ condition. And the negative charges injected from the ground will move quickly to meet the front edge of the positive charge peak, due to the DC offset' effects. However the negative charges near the ground sides can be neutralised by the newly injected positive charges, making the negative charge peak distorts sharply with the time. This becomes insignificant when the DC offset is high, as the holes' peak moves faster due to the mobility's field-dependant effects (Fig2).

\section{CONCLUSION}

Space charge within LDPE under the combined AC and DC stress has been simulated using the bipolar charge transport model. The accumulation of space charge under AC stress can be weakened by the frequent reversal of the applied field, while the addition of the DC component can neutralise this effect, and even promote the charge gathering when the DC component exceeds a certain value. Besides, DC offsets within the combined stresses can also distort the charge distribution further, assisting the charge near the surface region to transport through into the bulk of the insulation.

All these above show that, unlike under AC conditions, the space charge under complex electric stresses should be paid more attention. Depending on the ratio of DC component, charge dynamics change significantly. The present work also indicates that experiments need to be done to validate the simulation. Further modifications on the model may be required to improve its accuracy through comparison with experimental data.

Although the current simulation is based on the parameters taken from a well know insulation, polyethylene, the charge dynamics obtained from the simulations may have significant implication on the other insulation systems such as oil impregnated paper/pressboard used in HVDC converter transformers where the combined electric fields exist.

\section{REFERENCES}

[1] Y. Li and T. Takada, "Progress in-Space Charge Measurement of Solid Insulating Materials in Japan," Ieee Electrical Insulation Magazine, vol. 10, pp. 16-28, Sep-Oct 1994.

[2] R. J. Fleming, "Space charge in polymers, particularly polyethylene," Brazilian Journal of Physics, vol. 29, pp. 280-294, Jun 1999.

[3] J. M. ALISON and R. M. HILL, A model for bipolar charge transport, trapping and recombination in degassed crosslinked polyethene vol. 27. Bristol, ROYAUME-UNI: Institute of Physics, 1994.

[4] S. L. Roy, P. Segur, G. Teyssedre, and C. Laurent, "Description of bipolar charge transport in polyethylene using a fluid model with a constant mobility: model prediction," Journal of Physics D: Applied Physics, vol. 37, pp. 298-305, 2004.

[5] E. Belgaroui, I. Boukhris, A. Kallel, G. Teyssedre, and C. Laurent, "A new numerical model applied to bipolar charge transport, trapping and recombination under low and high dc voltages," Journal of Physics D: Applied Physics, vol. 40, p. 6760, 2007.

[6] A. See, J. C. Fothergill, L. A. Dissado, and J. M. Alison, "Measurement of space-charge distributions in solid insulators under rapidly varying voltage using the high-voltage, high-speed pulsed electro-acoustic (PEA) apparatus," Measurement Science \& Technology, vol. 12, pp. 1227-1234, Aug 2001.

[7] G. Chen, M. Fu, X. Z. Liu, and L. S. Zhong, "ac aging and space-charge characteristics in low-density polyethylene polymeric insulation," Journal of Applied Physics, vol. 97, Apr 152005.

[8] J. M. Alison and R. M. Hill, "A model for bipolar charge transport in insulators," in Conduction and Breakdown in Solid Dielectrics, 1995. ICSD'95., Proceedings of the 1995 IEEE 5th International Conference on, 1995, pp. 319-323.

[9] S. Le Roy, G. Teyssedre, C. Laurent, and P. Segur, "Numerical modeling of space charge and electroluminescence in polyethylene under dc field," in Electrical Insulation and Dielectric Phenomena, 2002 Annual Report Conference on, 2002, pp. 172-175.

[10] S. L. Roy, G. Teyssedre, C. Laurent, G. C. Montanari, and F. Palmieri, "Description of charge transport in polyethylene using a fluid model with a constant mobility: fitting model and experiments," Journal of Physics D: Applied Physics, vol. 39, pp. 1427-1436, 2006.

[11] J. Zhao, G. Chen, and P. L. Lewin, "Investigation into the formation of charge packets in polyethylene: Experiment and simulation," Journal of Applied Physics, vol. 112, pp. 034116-034116-6, 2012.

[12] G. Chen and J. W. Zhao, "Observation of negative differential mobility and charge packet in polyethylene," Journal of Physics D-Applied Physics, vol. 44, Jun 12011.

[13] J. Zhao, Z. Xu, G. Chen, and P. Lewin, "Effect of field-dependent mobility on current density and dynamics of space charge in polyethylene," in Electrical Insulation and Dielectric Phenomena, 2009. CEIDP '09. IEEE Conference on, 2009, pp. 120-123.

[14] G. Chen, J. Zhao, S. Li, and L. Zhong, "Origin of thickness dependent dc electrical breakdown in dielectrics," Applied Physics Letters, vol. 100, p. 222904, 2012.

[15] J. Zhao, Z. Xu, G. Chen, and P. L. Lewin, "Numeric description of space charge in polyethylene under ac electric fields," Journal of Applied Physics, vol. 108, Dec 152010. 